Supporting Information

\title{
Solubility Measurements and Correlation of MBQ- 167 in Neat and Binary Solvent Mixtures
}

Jocelyn M. Jiménez Cruz, ,2.2 Cornelis P. Vlaar, ${ }^{2}$ Vilmalí López-Mejías ${ }^{13^{3 *}}$ and Torsten Stelzer ${ }^{12^{*}}$

Crystallization Design Institute, Molecular Sciences Research Center, University of Puerto Rico, San Juan, Puerto Rico 00926, United States

2Department of Pharmaceutical Sciences, University of Puerto Rico - Medical Sciences Campus, San Juan, Puerto Rico 00936, United States

${ }^{3}$ Department of Chemistry, University of Puerto Rico - Río Piedras Campus, San Juan, Puerto Rico 00931, United States

*E-mail: vilmali.lopez@upr.edu (V.L.-M.)

*E-mail: torsten.stelzer@upr.edu (T.S.) 


\section{Differential Scanning Calorimetry (DSC) and Thermogravimetric Analysis (TGA)}

DSC was performed to determine the onset melting temperature $\left(T_{\mathrm{m}, \mathrm{onset}}\right)$ and enthalpy of fusion $\left(\Delta H_{f u s}\right)$ of MBQ-167. A DSC from TA Instruments Inc. (Q2000) equipped with a RCS40 single-stage refrigeration system was employed to carry out these measurements. The calibration of the DSC was conducted with an indium standard $\left(T_{\mathrm{m}}=429.75 \mathrm{~K}\right.$ and $\left.\Delta H_{f u s}=28.54 \mathrm{~J} / \mathrm{g}\right)$. Approximately $2.600 \mathrm{mg}$ of the microcrystalline sample was weighed using a XP26 microbalance from Mettler Toledo $( \pm 0.002 \mathrm{mg}$ ) and placed on Tzero aluminum pans that were hermetically sealed. Samples were equilibrated at $298.15 \mathrm{~K}$ for $5 \mathrm{~min}$ before heating to $523.15 \mathrm{~K}$ under a $\mathrm{N}_{2}$ atmosphere $(50 \mathrm{~mL} / \mathrm{min})$ at a rate of $5.0 \mathrm{~K} / \mathrm{min}$ and a temperature accuracy of $0.1 \mathrm{~K}$. A total of two $(n=2)$ thermograms were analyzed using the TA Universal Analysis 2000 software (version 4.5A). A representative thermogram of MBQ-167 is shown in Figure S1. The enthalpy of fusion $(\mathrm{J} / \mathrm{g})$ represents the area under the curve. To convert the value in $\mathrm{kJ} / \mathrm{mol}$, the enthalpy of fusion in J/g was multiplied by the molecular weight (MW) of MBQ-167 (MW = $338.41 \mathrm{~g} / \mathrm{mol}$ )' and divided by 1,000 . The average of the $\Delta H_{\text {fus }}$ and $T_{\mathrm{m}, \text { onset }}$ obtained for MBQ167 were determined as $30.1 \pm 0.1 \mathrm{~kJ} / \mathrm{mol}$ and $425.63 \pm 0.02 \mathrm{~K}$ as indicated in Table S1. The average value of $T_{\mathrm{m}, \text { onset }}$ was employed to determine the correlated mole fraction solubility $\left(x_{1}^{c a l}\right)$ using the $\lambda \mathrm{h}$ model equation.

Thermographs were recorded in a TGA from TA Instruments Inc. (Q500) calibrated with calcium oxalate monohydrate $\left(\mathrm{CaC}_{2} \mathrm{O}_{4} \cdot \mathrm{H}_{2} \mathrm{O}\right)$ standard to determine if MBQ-167 degrades before melting. About $1.400 \mathrm{mg}$ of the microcrystalline sample were equilibrated at $298.15 \mathrm{~K}$ for $5 \mathrm{~min}$ before heating to $673.15 \mathrm{~K}$ under a $\mathrm{N}_{2}$ atmosphere $(60 \mathrm{~mL} / \mathrm{min})$ at a rate of $5.0 \mathrm{~K} / \mathrm{min}$ and a temperature accuracy of $0.1 \mathrm{~K}$. Data was analyzed with the TA Universal Analysis 2000 software (version 4.5A). TGA reveals that MBQ-167 starts to decompose after the melting temperature $(425.63 \mathrm{~K})$. The thermograms shows that the sample begins to decompose at $\sim 458 \mathrm{~K}$ and ends at $\sim 565 \mathrm{~K}$ with a mass loss of $\sim 98.4 \mathrm{wt}$. \% (Figure S1 bottom). 


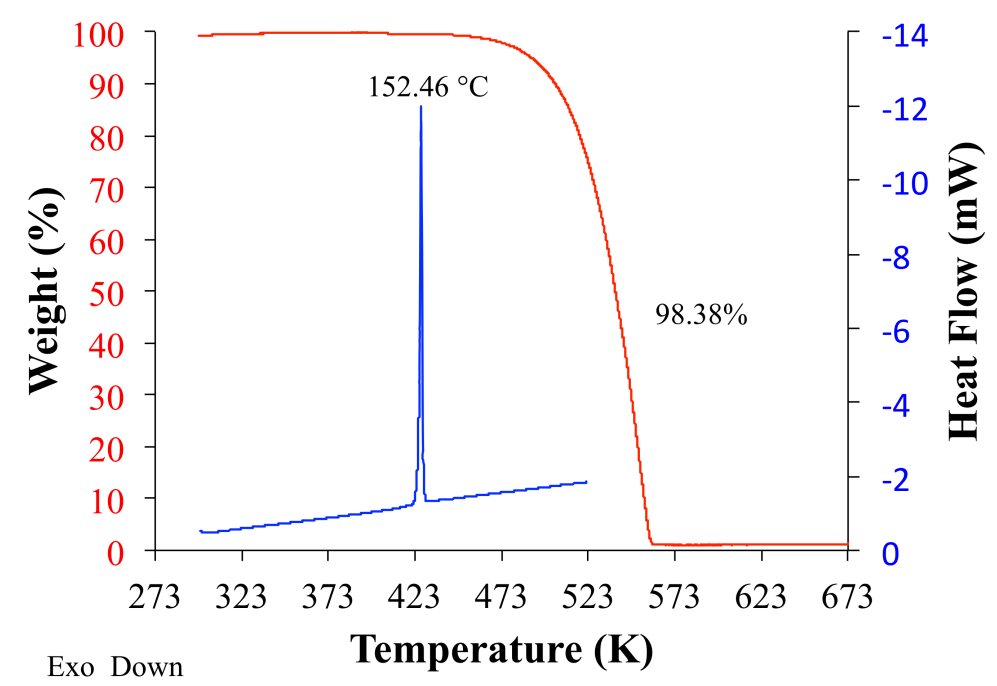

Figure S1. Representative DSC (blue) and TGA (red) thermograms of MBQ-167.

Table S1. Thermodynamics properties of MBQ-167 at a pressure $(p), p=101.3 \mathrm{kPa}$.

$\begin{array}{ccc} & T_{\mathrm{m}, \text { one }} / \mathrm{K} & \Delta H_{\text {fus }} / \mathrm{kJ} \cdot \mathrm{mol}^{-1} \\ & 425.64 & 30.0 \\ & 425.61 & 30.2 \\ \text { Average }(\mathrm{n}=2) & 425.63 & 30.1 \\ \text { STD } & 0.02 & 0.1\end{array}$

Standard uncertainty $u$ is $u(T)=0.02 \mathrm{~K}$ and $u\left(\Delta H_{f u s}\right)=0.1 \mathrm{~kJ} \cdot \mathrm{mol}^{-1}$. Relative standard uncertainty $u_{r}$ is $u(p)=0.1$.

To the best of our knowledge, no prior thermodynamic data has been reported for MBQ$167 \cdot \cdot^{2}$

\section{Solubility Measurements}

To determine the solubility of MBQ-167 (1) in eight neat solvents (acetonitrile, 1-butanol, 2-butanol, ethanol, ethyl acetate, methanol, 1-propanol, and 2-propanol) and two binary mixtures [(ethyl acetate (2) + heptane (3) and ethanol (2) + water (3)], the polythermal method was employed using a Crystal16 ${ }^{\mathrm{TM}}$ multiple reactor system from Technobis Crystallization Systems. The suspensions were prepared in $2 \mathrm{~mL}$ sealed glass vials (Fisher Scientific) at predetermined 
concentrations. A microbalance (XP26, $\pm 0.002 \mathrm{mg})$ and an analytical balance (MS104S, \pm 0.1 $\mathrm{mg}$ ), both from Mettler Toledo, were used to weigh the solute and solvents, respectively. While the suspensions were agitated at $700 \mathrm{rpm}$ using rare earth magnetic stir bars, they were heated from 278.15 to $333.15 \mathrm{~K}$ at rate of $0.1 \mathrm{~K} / \mathrm{min}$. Assuming that dissolution kinetics can be neglected, ${ }^{3}$ the clear point was determined by monitoring the transmission of the light source through the suspensions using the CrystalClear software (version 1.0.1.614). The uncertainty of the saturation temperature measurements is within $\pm 0.1 \mathrm{~K}$.

Before the solubility measurements, the heating rate applied $(0.1 \mathrm{~K} / \mathrm{min})$ was validated by comparing the experimental and correlated solubility data run using a higher rate at $0.3 \mathrm{~K} / \mathrm{min}$ (Figure S2). As shown, there is no significant difference between the two heating rates employed demonstrating that quasi solid-liquid equilibrium has been achieved. ${ }^{+6}$

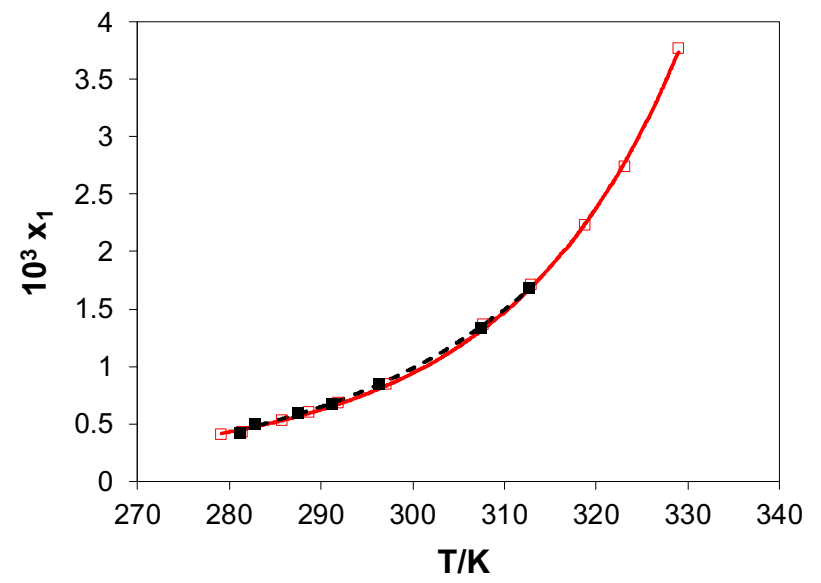

Figure S2. Experimental and correlated solubility data of MBQ-167 in ethanol at different heating rates. $\square, 0.1 \mathrm{~K} / \mathrm{min} ; \mathbf{\square}, 0.3 \mathrm{~K} / \mathrm{min}$; -, calculated using the modified Apelblat equation.

\subsection{Solubility Scatter Plots}

Figure S3 shows the correlated and experimentally derived solubility data of MBQ-167 in the neat solvents using the $\lambda \mathrm{h}$ model equation. Figures S4 and S5 illustrate the correlated and 
experimentally derived solubility data of MBQ-167 in binary solvent mixtures utilizing the modified Apelblat equation (left side) and the $\lambda \mathrm{h}$ model equation (right side).
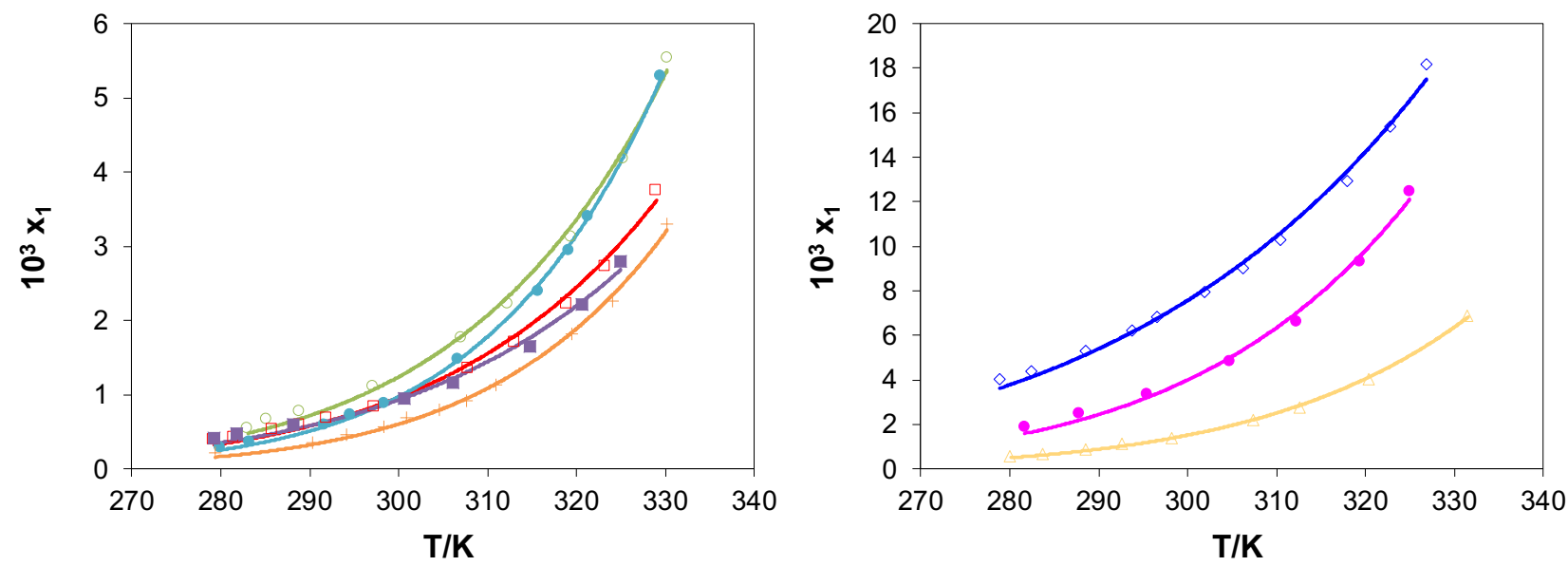

Figure S3. Experimental and correlated solubility data of MBQ-167 in neat solvents, from top to bottom; (on the left), ○, 1-propanol; •, 2-butanol; $\square$, ethanol; $\square$, methanol; +, 2-propanol; (on the right), $\diamond$, ethyl acetate; •, acetonitrile; $\triangle 1$-butanol - , calculated using the $\lambda \mathrm{h}$ equation. $x_{1}$ represents the mole fraction solubility of MBQ-167 and $T$ the temperature in Kelvin (K).
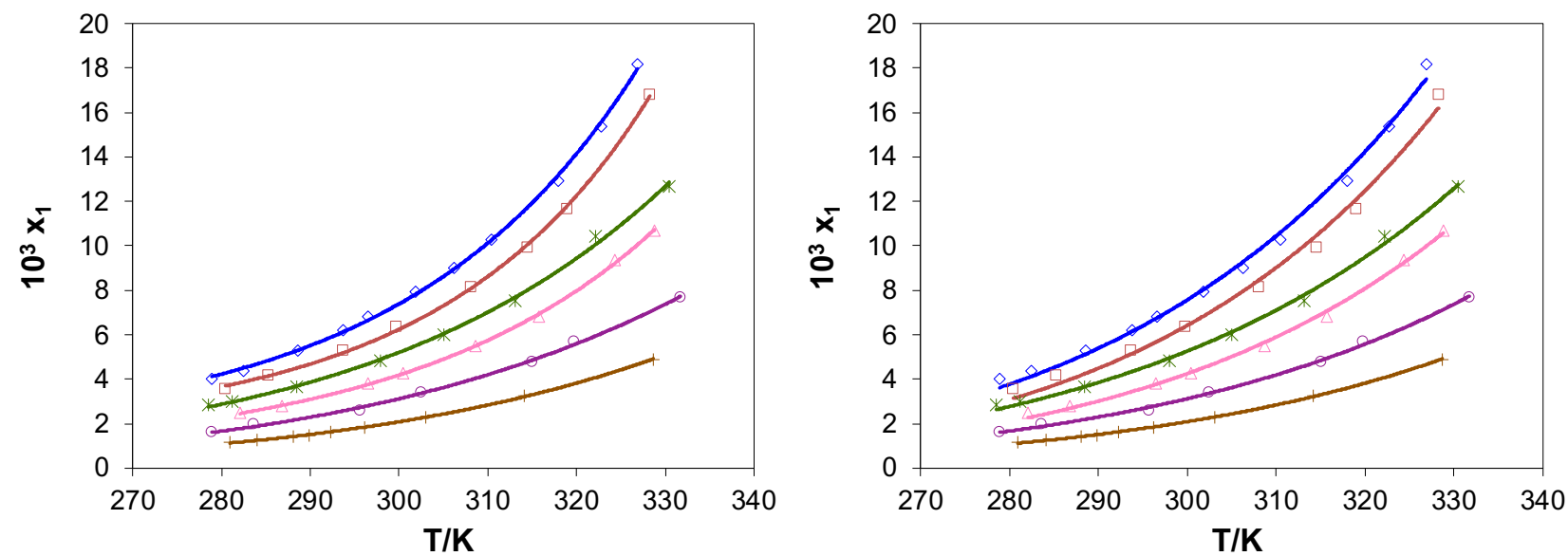

Figure S4. Experimental and correlated solubility data of MBQ-167 (1) in ethyl acetate (2) + heptane (3) solvent mixtures. $\diamond, W_{3}=0$ (heptane); $\square, W_{3}=0.08 ; *, W_{3}=0.16 ; \Delta, W_{3}=0.23, \circ, W_{3}=$ $0.34 ;+, W_{3}=0.43 ;-$, calculated using the modified Apelblat equation (left) and $\lambda \mathrm{h}$ equation (right). $W_{3}$ is the mass fraction of heptane (3) in ethyl acetate (2) + heptane (3) binary mixture. $x_{1}$ represents the mole fraction solubility of MBQ-167 and $T$ the temperature in Kelvin (K). 

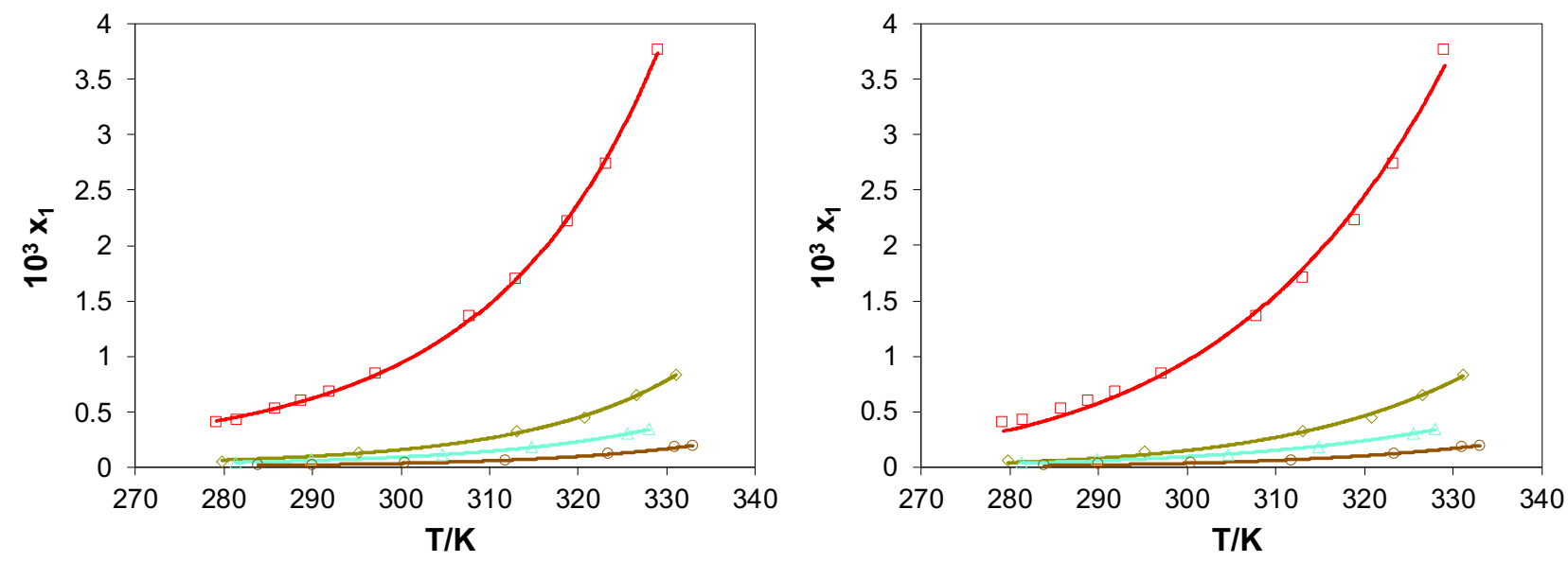

Figure S5. Experimental and correlated solubility data of MBQ-167 (1) in binary ethanol (2) + water (3) solvent mixtures. $\square, W_{3}=0 ; \diamond, W_{3}=0.24 ; \Delta, W_{3}=0.36 ; \circ, W_{3}=0.46$ (water); -, calculated using the modified Apelblat equation (left) and $\lambda \mathrm{h}$ equation (right). $W_{3}$ is the mass fraction of water (3) in ethanol (2) + water (3) binary mixture. $x_{1}$ represents the mole fraction solubility of MBQ-167 and $T$ the temperature in Kelvin (K).

\subsection{Solubility Surface Plots}

To generate the surface plots, Origin (OriginLab Corporation, version 9.7.0.188) was used with the Random Kriging Correlation method for $x y z$ gridding. The number of rows and columns for the gridding were selected based on the number of different solvent compositions within each specific system of binary solvent mixtures and the maximum number of data points. The minimum and maximum points were set to 10 and 100, respectively, whereas smoothing and search radius were set to one and two, respectively. Figures S6 and S7, illustrate the surface plots for the correlated solubility data of MBQ-167 in binary solvent mixtures using the $\lambda \mathrm{h}$ model equation. Surface plots correlated using the modified Apelblat equation can be found within the manuscript. 


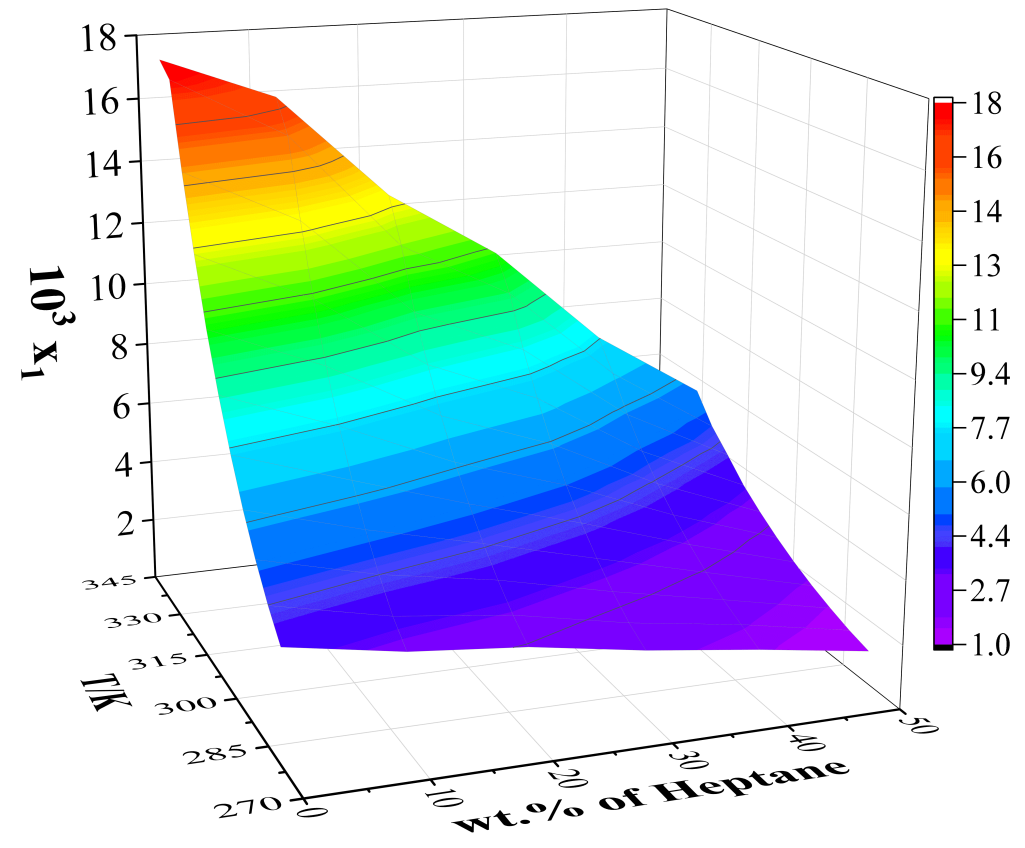

Figure S6. Surface plot for the solubility of MBQ-167 (1) in binary solvent system ethyl acetate (2) + heptane (3) correlated with the $\lambda \mathrm{h}$ equation. $x_{1}$ represents the mole fraction solubility of MBQ-167 and $T$ the temperature in Kelvin (K).

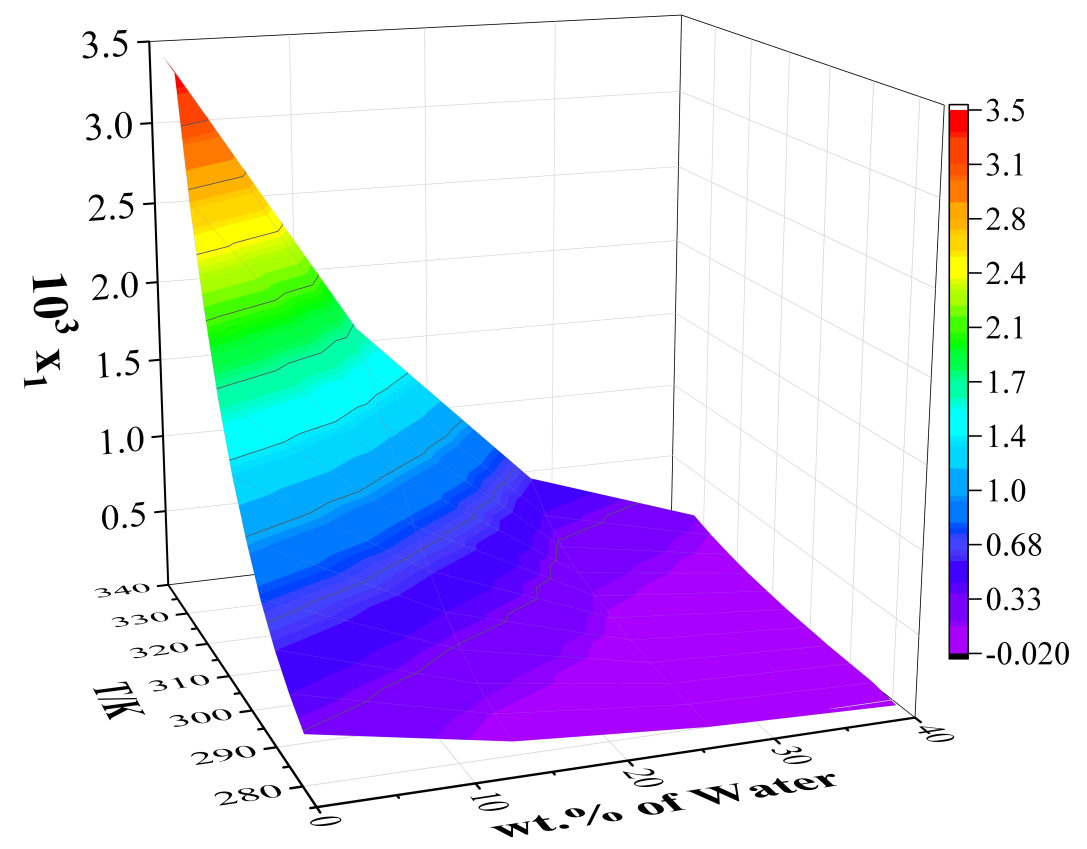

Figure S7. Surface plot for the solubility of MBQ-167 (1) in binary solvent system ethanol (2) + water (3) correlated with the $\lambda \mathrm{h}$ equation. $x_{1}$ represents the mole fraction solubility of MBQ167 and $T$ the temperature in Kelvin $(\mathrm{K})$. 


\section{Powder X-ray Diffraction (PXRD) Analysis}

Diffractograms were collected at $300 \mathrm{~K}$ using a Rigaku XtaLAB SuperNova single microfocus $\mathrm{Cu}-\mathrm{K} \alpha$ radiation $(\lambda=1.5417 \AA$ ) source equipped with a HyPix3000 X-ray detector operating at $50 \mathrm{kV}$ and $1 \mathrm{~mA}$ in transmission mode. Microcrystalline samples were placed in MiTeGen micro loops with the aid of a slight amount of paratone oil. Diffractograms were collected over an angular range of $6-60^{\circ}$ in $2 \theta$ with a step size of $0.01^{\circ}$. To perform the diffraction experiment, a Gandolfi move for powders with an exposure of $120 \mathrm{~s}$ and a detector distance of $100 \mathrm{~mm}$ was employed. Data was analyzed within the CrysAlis ${ }^{\text {гео }}$ software (v. 1.171.39.46). MBQ-167 was analyzed by PXRD previous to the solubility measurements and the solid-state was confirmed. Once the experiments were concluded, the resulting suspensions were analyzed by PXRD to confirm that the recrystallized material conformed to the only known solid form of MBQ-167 (Figures S8-S24). PXRDs display shades of color corresponding to the mole fraction solubility displayed in the solubility curves (e.g. pink represents acetonitrile as a solvent). The darker the shades of a color within the diffractograms, the higher the mole fraction solubility used to crystallize the resulting solid.

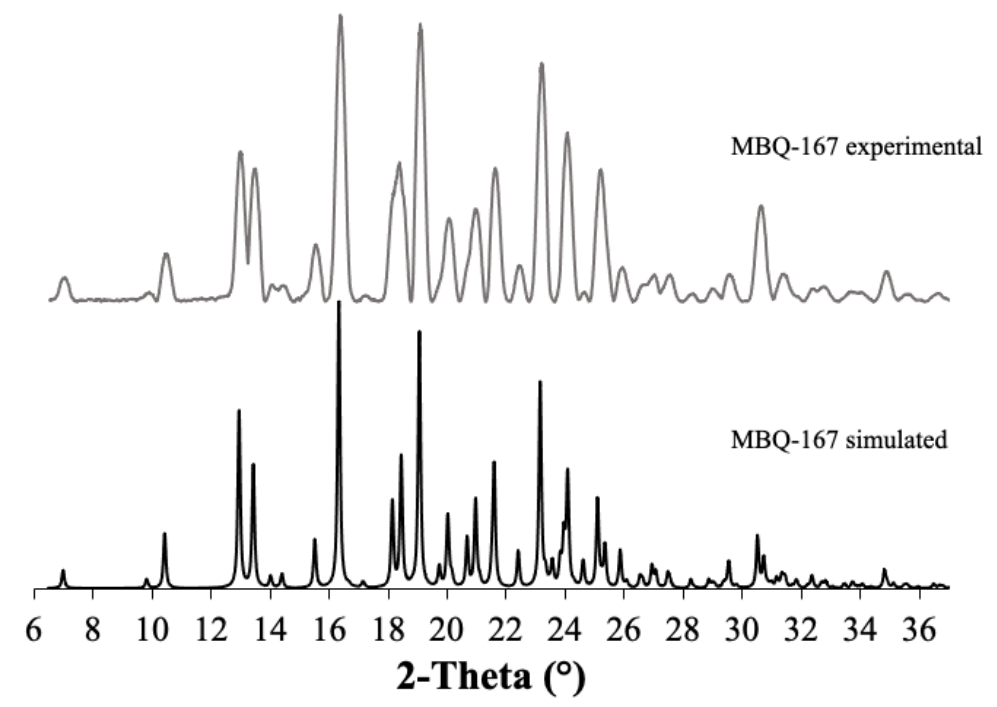

Figure S8. Representative powder X-ray diffractograms of the "as received" compound (grey) compared to the simulated diffractogram of MBQ-167 from the solved crystal structure at $300 \mathrm{~K}$ (black). 


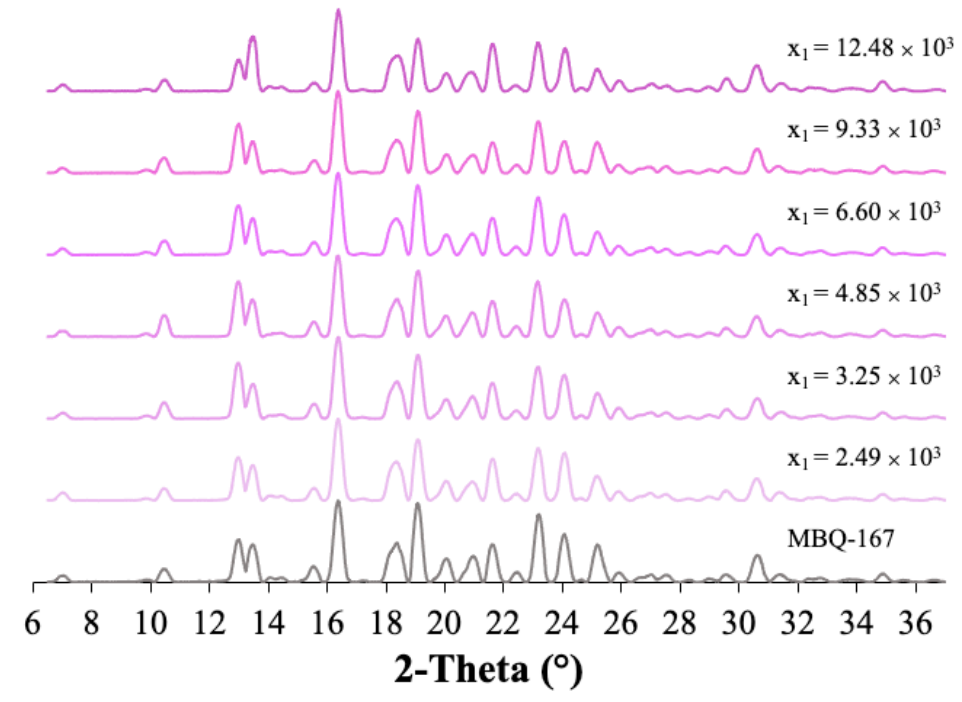

Figure S9. Representative powder X-ray diffractograms of MBQ-167 recrystallized from acetonitrile (shades of pink) at different mole fractions compared to the "as received" MBQ-167 (grey).

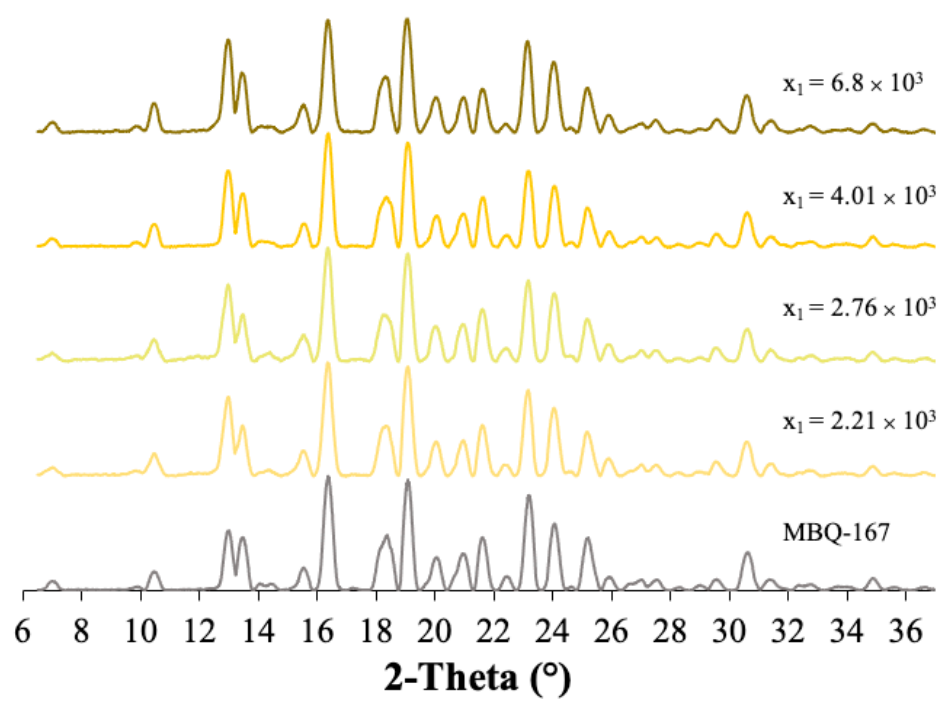

Figure S10. Representative powder X-ray diffractograms of MBQ-167 recrystallized from 1butanol (shades of yellow) at different mole fractions compared to the "as received" MBQ-167 (grey). 


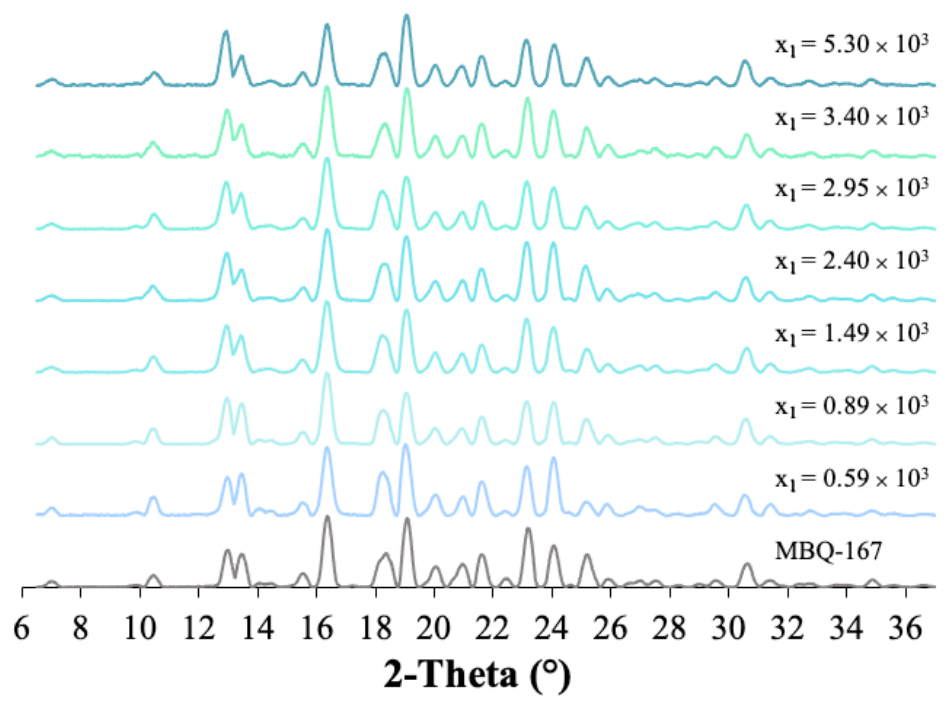

Figure S11. Representative powder X-ray diffractograms of MBQ-167 recrystallized from 2butanol (shades of blue) at different mole fractions compared to the "as received" MBQ-167 (grey).

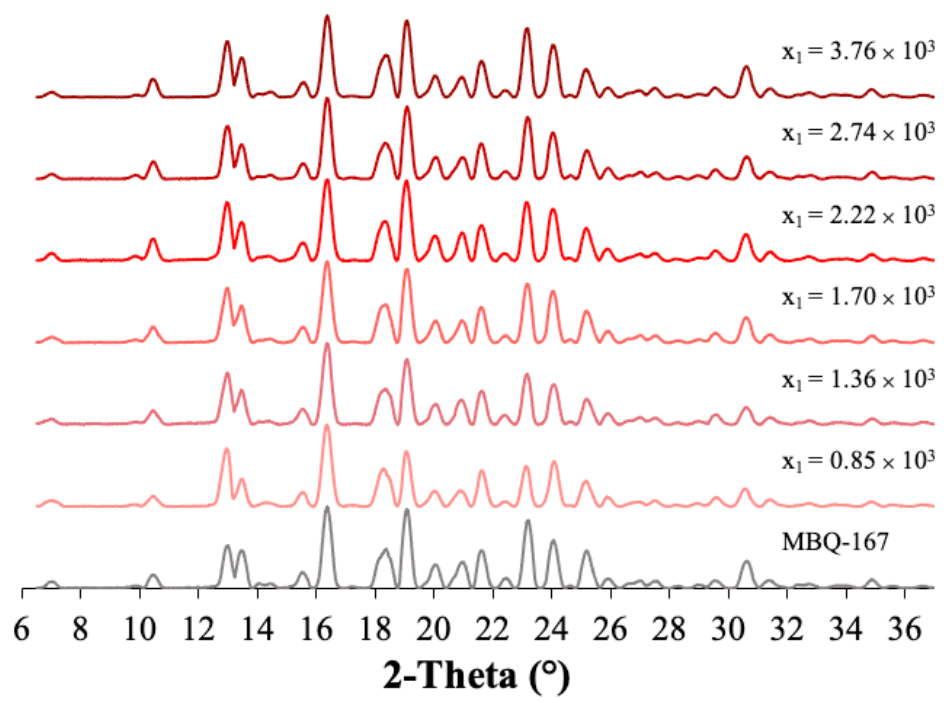

Figure S12. Representative powder X-ray diffractograms of MBQ-167 recrystallized from ethanol (shades of red) at different mole fractions compared to the "as received" MBQ-167 (grey). 


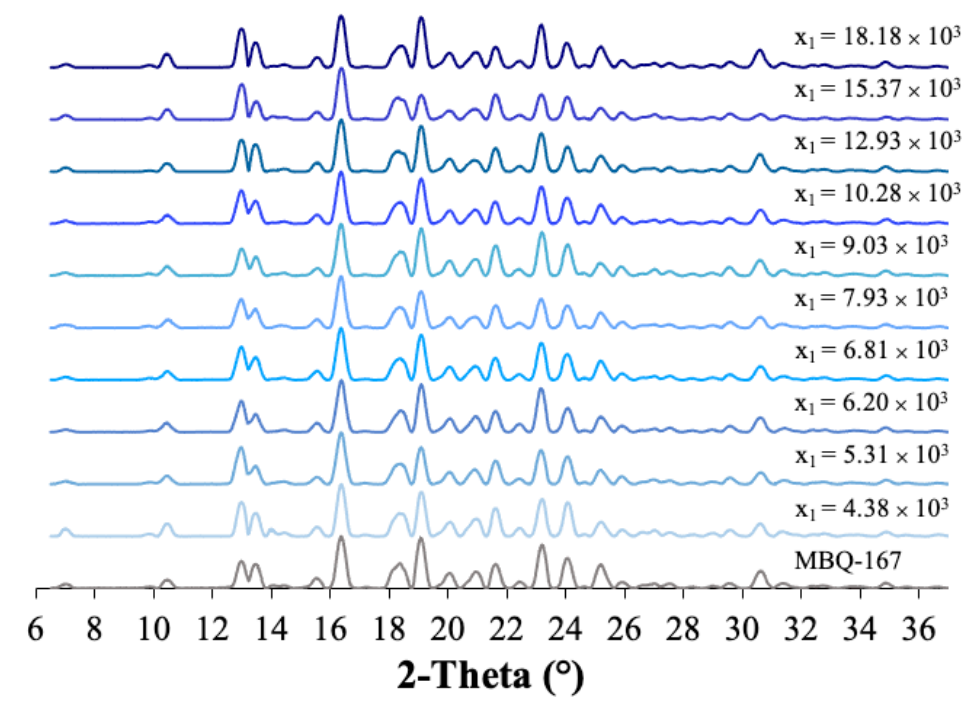

Figure S13. Representative powder X-ray diffractograms of MBQ-167 recrystallized from ethyl acetate (shades of blue) at different mole fractions compared to the "as received" MBQ-167 (grey).

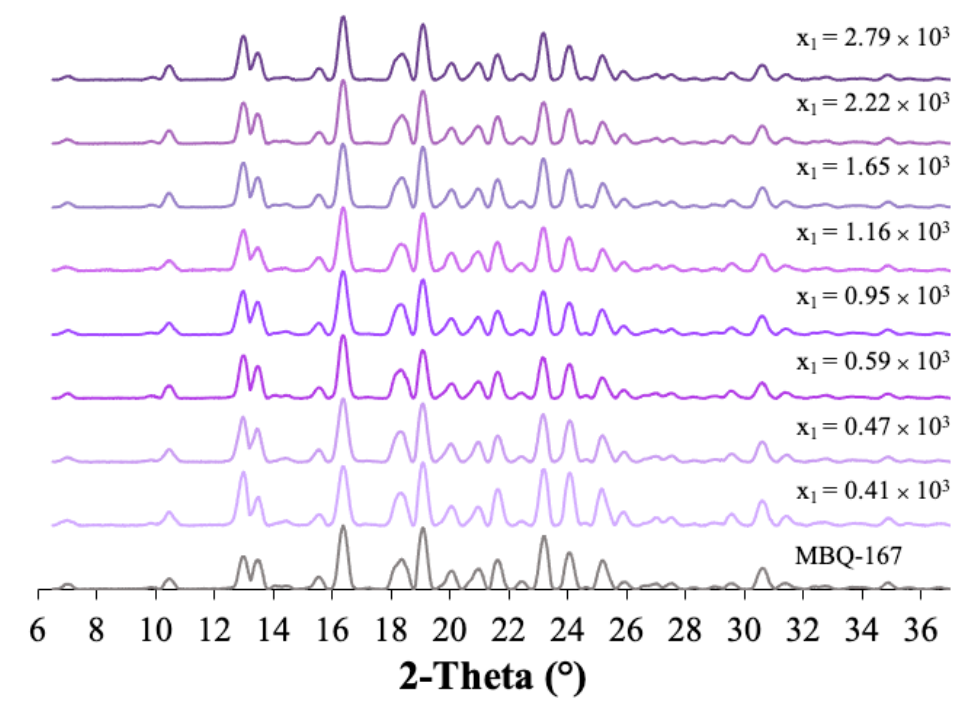

Figure S14. Representative powder X-ray diffractograms of MBQ-167 recrystallized from methanol (shades of purple) at different mole fractions compared to the "as received" MBQ-167 (grey). 


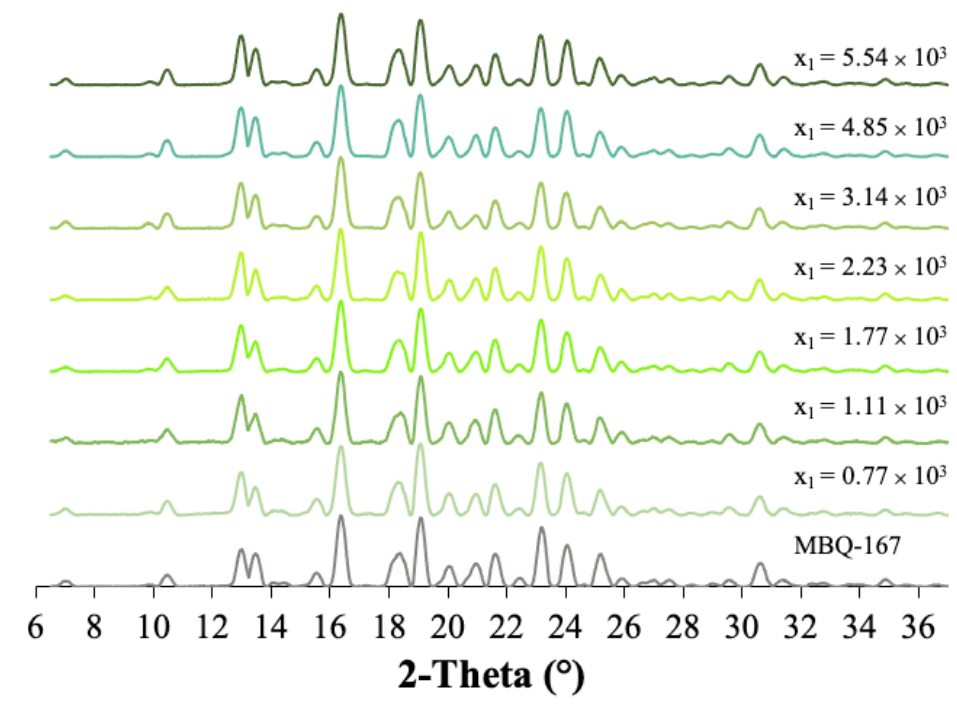

Figure S15. Representative powder X-ray diffractograms of MBQ-167 recrystallized from 1propanol (shades of green) at different mole fractions compared to the "as received" MBQ-167 (grey).

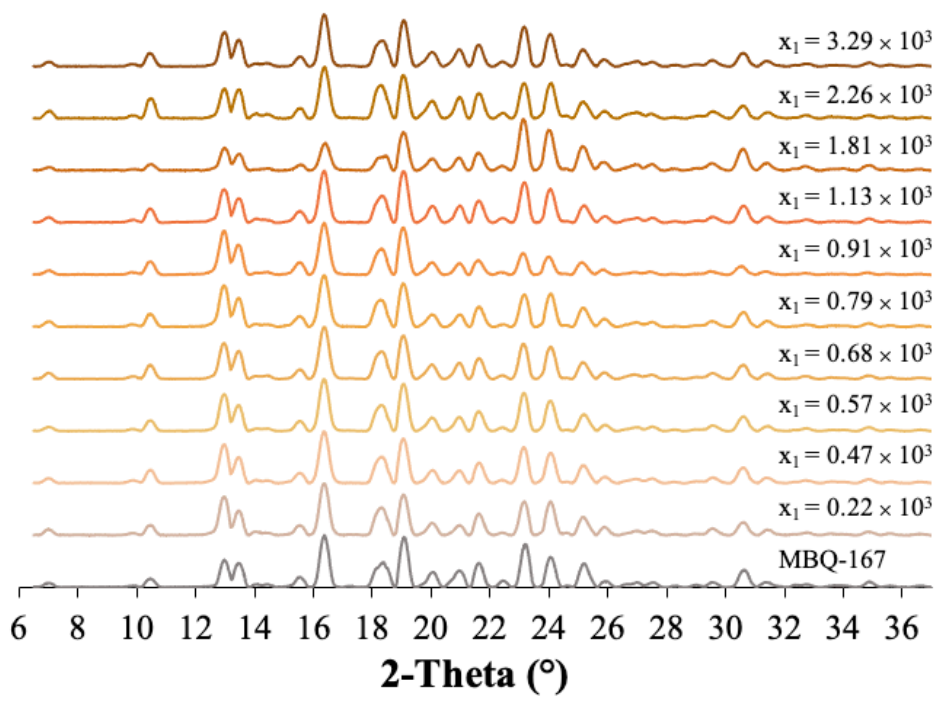

Figure S16. Representative powder X-ray diffractograms of MBQ-167 recrystallized from 2propanol (shades of orange) at different mole fractions compared to the "as received" MBQ-167 (grey). 


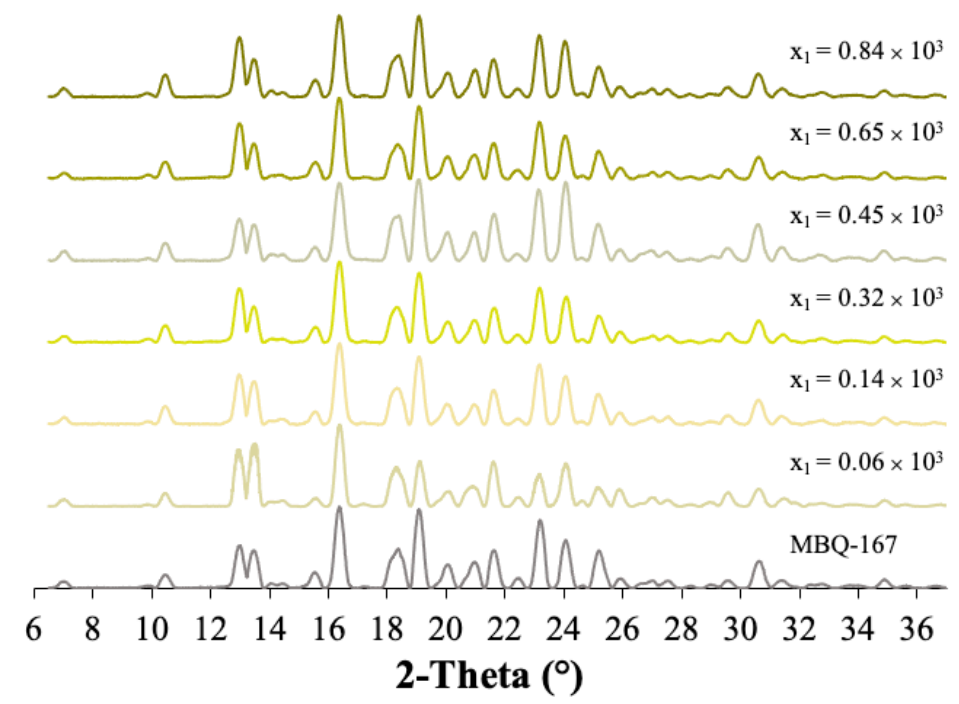

Figure S17. Representative powder X-ray diffractograms of MBQ-167 (1) crystals obtained in ethanol (2) + water (3) with $W_{3}=0.24$ binary solvent system (bottom to top); compared to the "as received" MBQ-167 (grey). $W_{3}$ is the mass fraction of water (3) in the ethanol (2) + water (3) binary solvent mixture.

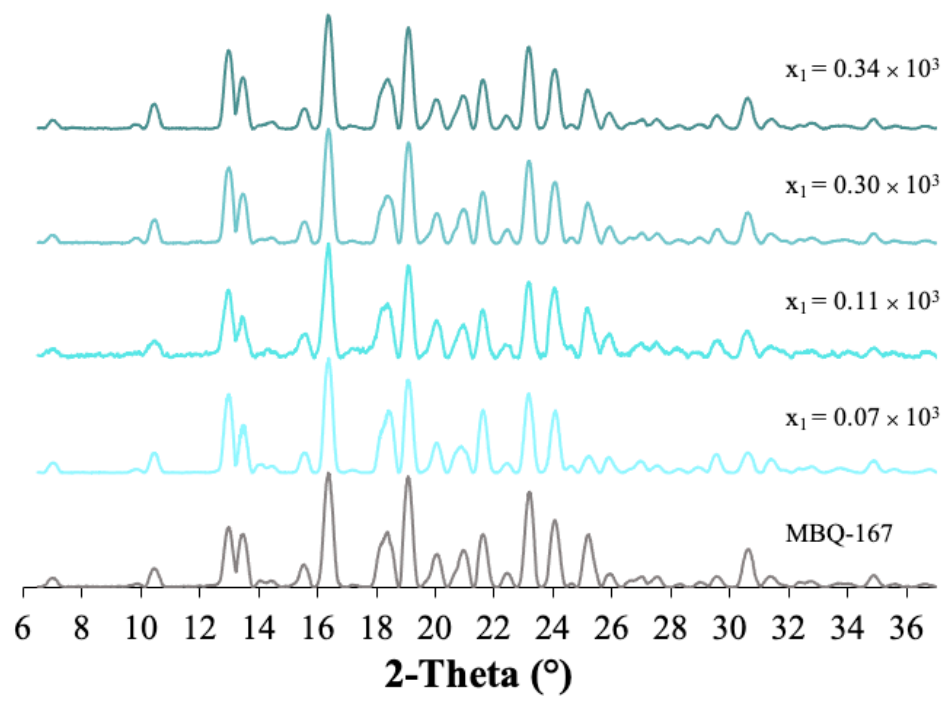

Figure S18. Representative powder X-ray diffractograms of MBQ-167 (1) crystals obtained in ethanol (2) + water (3) with $W_{3}=0.36$ binary solvent system (bottom to top); compared to the "as received" MBQ-167 (grey). $W_{3}$ is the mass fraction of water (3) in the ethanol (2) + water (3) binary solvent mixture. 


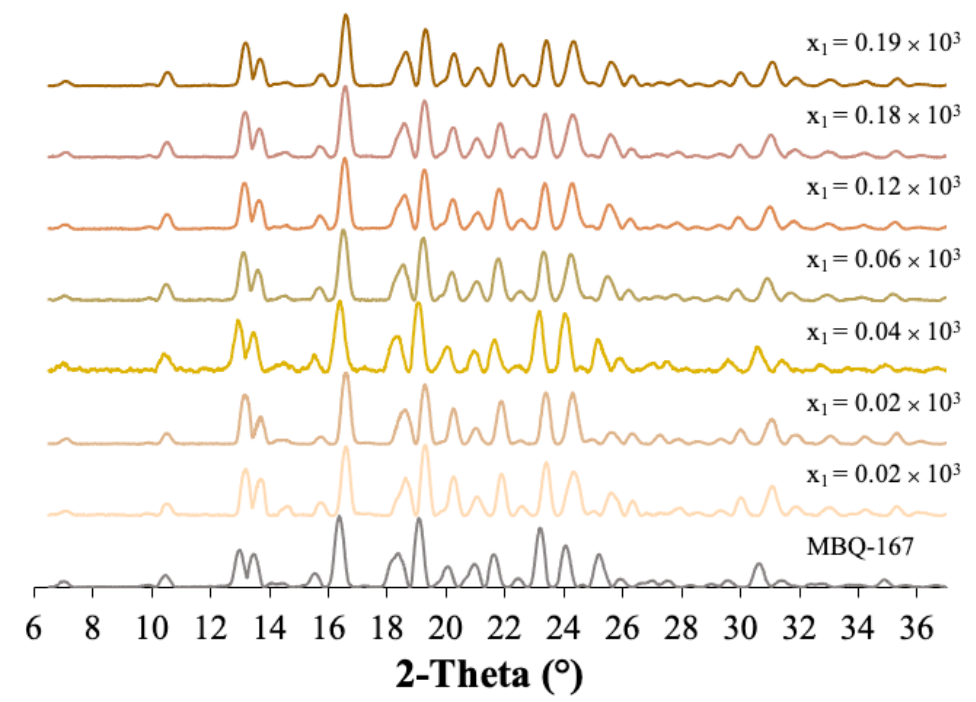

Figure S19. Representative powder X-ray diffractograms of MBQ-167 (1) crystals obtained in ethanol (2) + water (3) with $W_{3}=0.46$ binary solvent system (bottom to top); compared to the "as received" MBQ-167 (grey). $w_{3}$ is the mass fraction of water (3) in the ethanol (2) + water (3) binary solvent mixture.

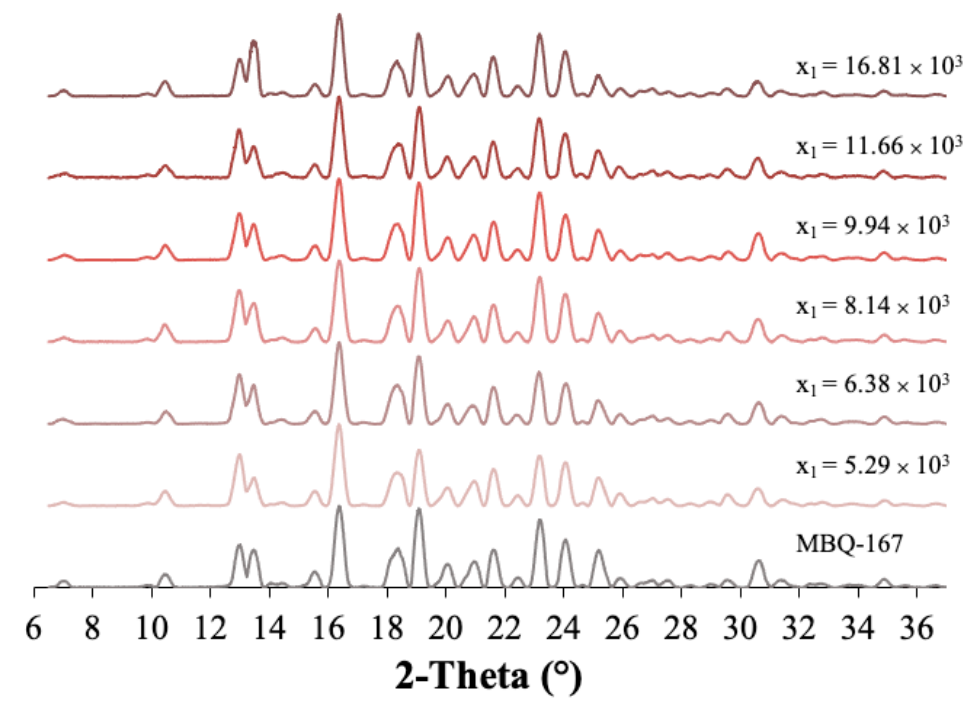

Figure S20. Representative powder X-ray diffractograms of MBQ-167 (1) crystals obtained in ethyl acetate (2) + heptane (3) with $W_{3}=0.08$ binary solvent system (bottom to top); compared to the "as received" MBQ-167 (grey). $W_{3}$ is the mass fraction of heptane (3) in the ethyl acetate (2) + heptane (3) binary solvent mixture. 


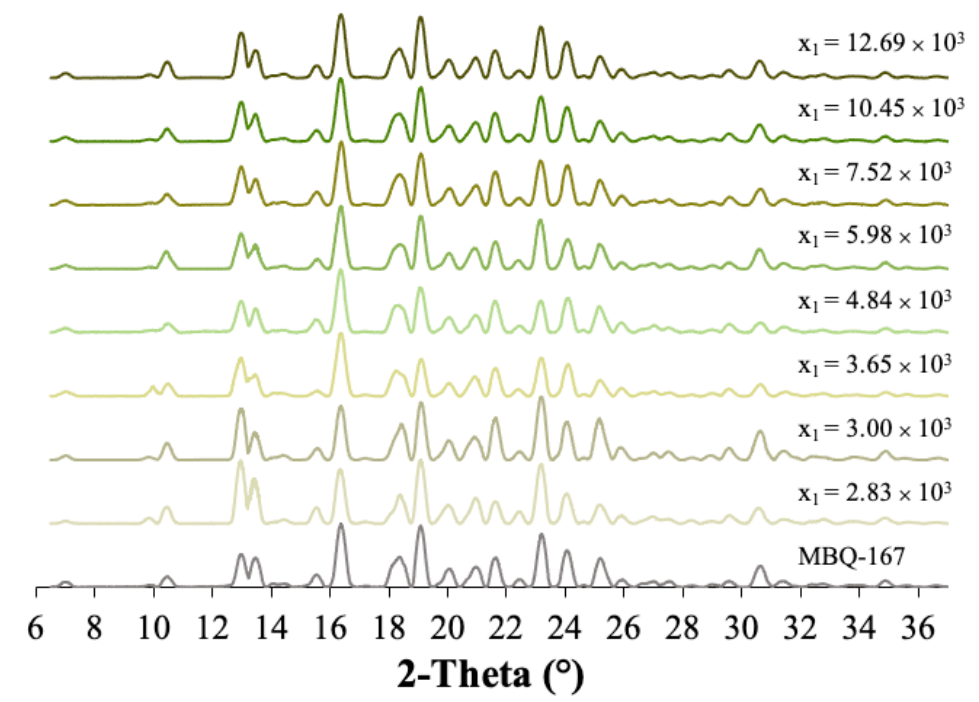

Figure S21. Representative powder X-ray diffractograms of MBQ-167 (1) crystals obtained in ethyl acetate (2) + heptane (3) with $W_{3}=0.16$ binary solvent system (bottom to top); compared to the "as received" MBQ-167 (grey). $W_{3}$ is the mass fraction of heptane (3) in the ethyl acetate (2) + heptane (3) binary solvent mixture.

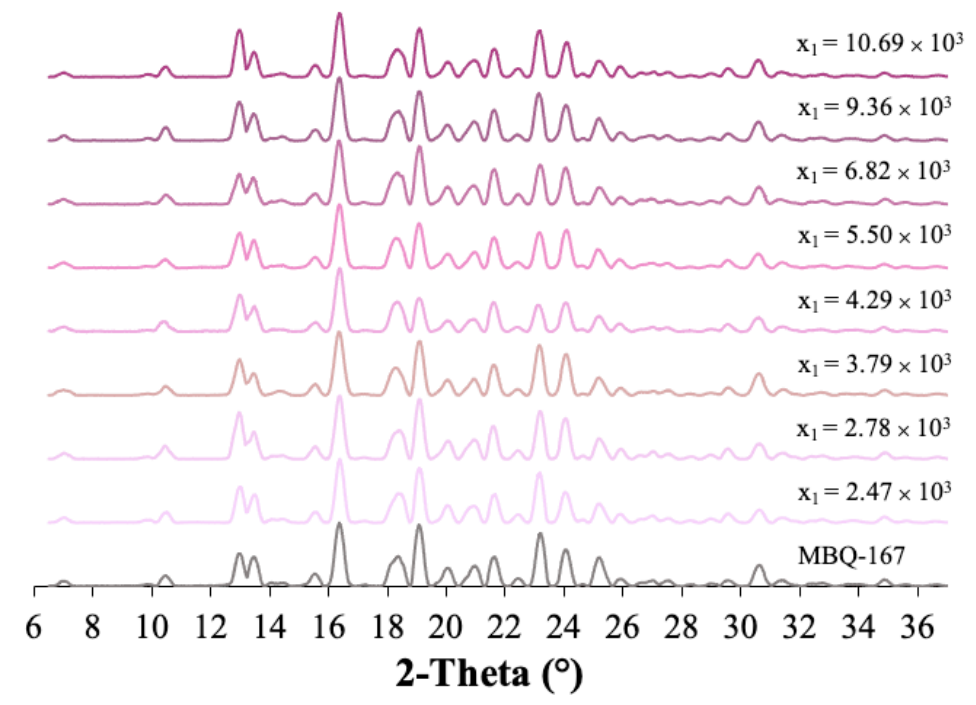

Figure S22. Representative powder X-ray diffractograms of MBQ-167 (1) crystals obtained in ethyl acetate (2) + heptane (3) with $W_{3}=0.23$ binary solvent system (bottom to top); compared to the "as received" MBQ-167 (grey). $W_{3}$ is the mass fraction of heptane (3) in the ethyl acetate (2) + heptane (3) binary solvent mixture. 


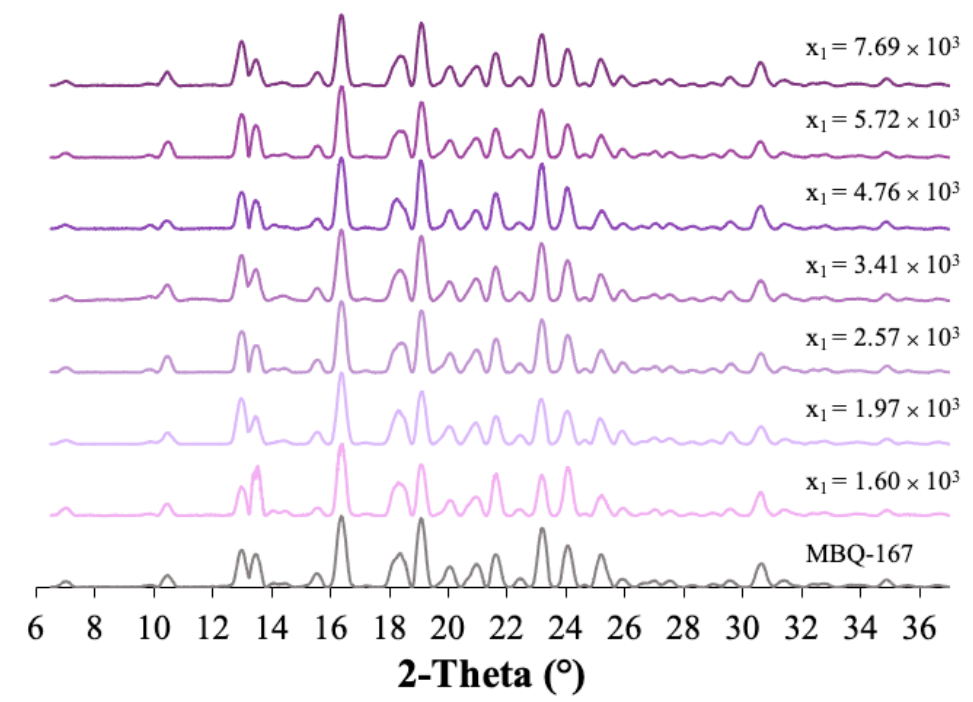

Figure S23. Representative powder X-ray diffractograms of MBQ-167 (1) crystals obtained in ethyl acetate (2) + heptane (3) with $W_{3}=0.34$ binary solvent system (bottom to top); compared to the "as received" MBQ-167 (grey). $W_{3}$ is the mass fraction of heptane (3) in the ethyl acetate (2) + heptane (3) binary solvent mixture.

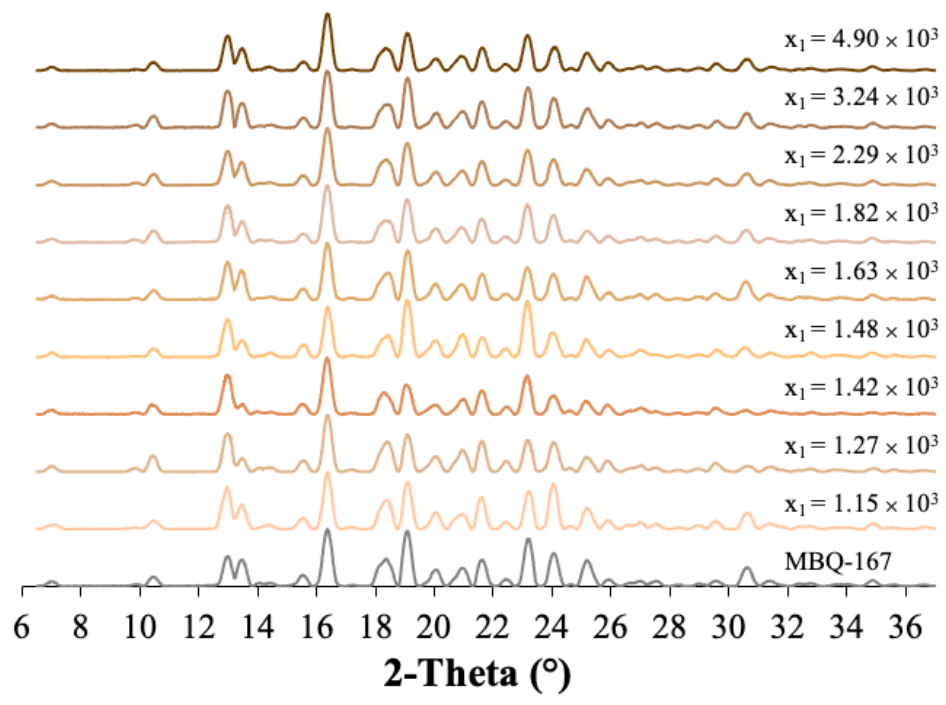

Figure S24. Representative powder X-ray diffractograms of MBQ-167 (1) crystals obtained in ethyl acetate (2) + heptane (3) with $W_{3}=0.43$ binary solvent system (bottom to top); compared to the "as received" MBQ-167 (grey). $W_{3}$ is the mass fraction of heptane (3) in the ethyl acetate (2) + heptane (3) binary solvent mixture. 


\section{$4 \quad$ References}

(1) Vlaar, C. P.; Dharmawardhane Flanagan, S.; Hernandez-O’Farrill, E.; Castillo-Pichardo, L. 1,5-Disubstituted 1,2,3-Triazoles Are Inhibitores of RAC/CDC42 GTPases. US 9,981,980 B2, 2018.

(2) Humphries-Bickley, T.; Castillo-Pichardo, L.; Hernandez-O'Farrill, E.; Borrero-Garcia, L. D.; Forestier-Roman, I.; Gerena, Y.; Blanco, M.; Rivera-Robles, M. J.; Rodriguez-Medina, J. R.; Cubano, L. A.; Vlaar, C. P.; Dharmawardhane, S. Characterization of a Dual Rac/Cdc42 Inhibitor MBQ-167 in Metastatic Cancer. Mol. Cancer Ther. 2017, 16, 805-818.

(3) Vellema, J.; Hunfeld, N. G. M.; Akker, H. E. A. Van Den; Horst, J. H. Avoiding Crystallization of Lorazepam during Infusion. Eur. J. Pharm. Sci. 2011, 44, 621-626.

(4) Vázquez Marrero, V. R.; Piñero Berríos, C.; De Dios Rodríguez, L.; Stelzer, T.; LópezMejías, V. In the Context of Polymorphism: Accurate Measurement, and Validation of Solubility Data. Cryst. Growth Des. 2019, 19, 4101-4108.

(5) Zorrilla-Veloz, R. I.; Stelzer, T.; López-Mejías, V. Measurement and Correlation of the Solubility of 5-Fluorouracil in Pure and Binary Solvents. J. Chem. Eng. Data 2018, 63, 3809_ 3817.

(6) George De la Rosa, M. V.; Santiago, R.; Malavé Romero, J.; Duconge, J.; Monbaliu, J.C.; López-Mejías, V.; Stelzer, T. Solubility Determination and Correlation of Warfarin Sodium 2-Propanol Solvate in Pure, Binary, and Ternary Solvent Mixtures. J. Chem. Eng. Data 2019, $64,1399-1413$. 